\title{
Thermomechanical Properties of Shape Memory Polymers of Polyurethane Series and their Applications
}

\author{
H. Tobushi, S. Hayashi*, A. Ikai** and H. Hara***
}

Department of Mechanical Engineering, Aichi Institute of Technology, 1247 Yachigusa, Yagusa-cho, Toyota 470-03, Japan

* High Polymer Research Laboratory, Mitsubishi Heavy Industries, Ltd., Nagoya Research

\& Development Center, 1 Takamichi, Iwatsuka-cho, Nakamura-ku, Nagoya 453, Japan

** Rinnai Co., Ltd. 2-26 Fukuzumi-cho, Nakagawa-ku, Nagoya 454, Japan

*** Graduate School, Aichi Institute of Technology, Japan

\begin{abstract}
Shape memory polymers of polyurethane series have been developed. These polymers have the following features. (1) They can be molded easily similar to conventional plastics, (2) their shape recovery temperature can be set arbitrarily, and (3) they can be colored as required because they are transparent, making a wide range of applications possible. In this paper, the basic characteristics of the stress-straintemperature-time relationship of shape memory polymers of the polyurethane series are investigated experimentally, and several applications of these polymers are introduced.
\end{abstract}

\section{INTRODUCTION}

The development of the applications of shape memory polymer (SMP) is drawing attention. Because SMP is light, high in shape recovery ability, easy to manipulate and economical as compared with shape memory alloy, its development is being actively promoted. The basic properties of shape memory alloy and SMP are shown in Table 1. In many SMPs, the phase transition temperature is around room temperature. Characteristics such as shape recoverability and shape fixity exist due to the difference between mechanical properties of materials above and below the phase transition temperature. SMPs utilizing these characteristics are being used as temperature sensors and actuators.

The polyurethane series of SMP has the features that (1) it can be molded by such methods as injection, extrusion and blowing, similar to conventional plastics, (2) it can be colored to any color as it is transparent and (3) the shape recovery temperature can be set anywhere between room temperature $\pm 50 \mathrm{~K}$, making a wide range of applications possible[1]-[5].

In this paper, the mechanical properties of SMP of the polyurethane series are examined. The basic characteristics of the stress-strain-temperature-time relationship are investigated experimentally for two kinds of materials which vary in elastic modulus at high temperature. Several applications of these polymers are introduced. 
Table 1 Comparison of SMA and SMP properties

\begin{tabular}{|c|c|c|}
\hline Materials & Ti Ni shape memory alloy & Shape memory polymer \\
\hline Recovery stress & $200-400 \mathrm{MPg}$ & $1-3 \mathrm{MPa}$ \\
\hline Recovery strain & $6 \%$ & $50-600 \%$ \\
\hline At low temp. & Soft $\left(E_{1}\right)$ & Hard $\left(E_{1}=100 E_{k}\right)$ \\
\hline At high temp. & Hard $\left(E_{k}=2 E_{1}\right)$ & Soft $\left(E_{h}\right)$ \\
\hline Derisity & $6-7 \mathrm{~g} / \mathrm{cm}^{3}$ & $1 \mathrm{~g} / \mathrm{cm}^{3}$ \\
\hline Phase transiormation & Martensitic, R-phase & Glass transition \\
\hline Temperature & $M_{n} M_{n} A_{n} A_{r}$ & $T_{8}$ \\
\hline Shaping & Difficult & Easy \\
\hline Cost & Expensive & Cheap \\
\hline Heat conductivity & High & Low \\
\hline
\end{tabular}

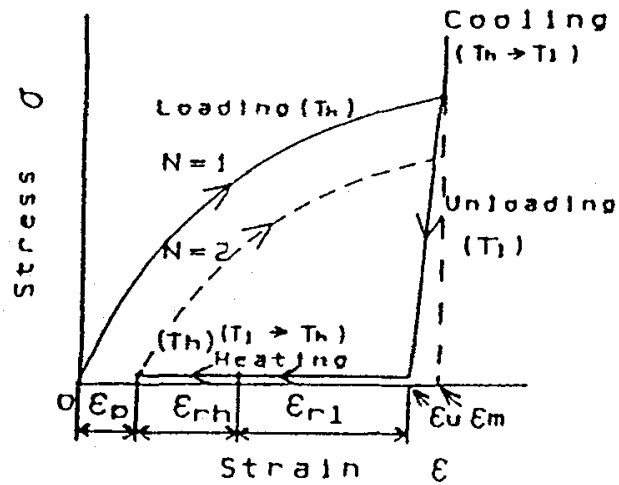

Fig.1 Stress-strain relationship in the thermomechanical cycling test with loading at high temperature

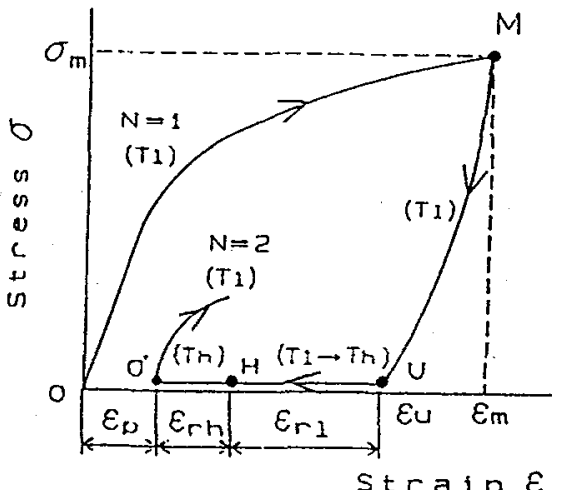

Fig.2 Stress-strain relationship in the thermomechanical cycling test with loading at low temperature

\section{EXPERIMENTAL METHOD}

Two SMPs of the polyurethane series, polyurethane of the polyester polyole series having high elastic modulus at high temperature and polyurethane of the polyether polyole series having low elastic modulus (Diary MM-4510 and 4520, respectively; produced by Mitsubishi Heavy Industries, Ltd., hereinafter referred to as material HE and material LE) were used. The glass transition temperature $T_{8}$ of both materials was about $318 \mathrm{~K}$.

The specimen was a plate of uniform shape. The dimensions of the testing area were $25 \mathrm{~mm}$ long, $1 \mathrm{~mm}$ thick and $5 \mathrm{~mm}$ wide.

The stress-strain relationship in the thermomechanical cycling test. with loading at high temperature is schematically shown in Fig.1. First, at the temperature $T_{h}$, the maximum strain $\varepsilon_{m}$ is applied at a constant strain rate of $50 \% / m i n$, and then, maintaining $\varepsilon_{m}$, the temperature is cooled to $T_{l}$. After holding at $T_{l}$ for 5 :minutes, it is unloaded. Under the no-load condition, it is heated from $T$ to $T_{h}$ in 5 minutes and held for time $t_{h}$ at $T h$. This thermomechanical cycle was repeated $N$ times.

The stress-strain relationship in the thermomechanical cycling test with loading at low temperature is schematically shown in Fig. 2. In this test, the specimen was first loaded up to the maximum strain $\varepsilon_{m}$ (Point $M$ ) at the constant strain rate of 
$50 \% / m i n$ at the temperature $T_{r}$ below $T_{\mathbf{g}}$, and then unloaded until no-load state was reached (Point $U$ ). Successively it was heated from the low temperature $T l$ to the high temperature $T_{h}$ (Point $\mathrm{H}$ ) in the no load state, and was held at $T_{h}$ for 5 minutes (Point $0^{\prime}$ ). Counting this thermomechanical cycle as one cycle, $N$ cycles were repeated.

These experiments were conducted by taking $T_{h}=338 \mathrm{~K}\left(=T_{g}+20 \mathrm{~K}\right)$ and $T_{l}=298 \mathrm{~K}\left(=T_{g}-20 \mathrm{~K}\right)$, and about $8 \mathrm{~K} / \mathrm{min}$ was given to the heating and cooling rate.

\section{EXPERIMENTAL RESULTS AND DISCUSSION}

In processing the experimental data, the stress and the strain were treated as nominal stress and nominal strain.

\subsection{Stress-Strain Curves}

The stress-strain curves obtained in the thermomechanical cycling test with loading at high temperature are shown for the case of maximum strain $\varepsilon_{m}=100 \%$ and $t_{h}=5 \mathrm{~min}$ in Fig. 3 . The curves are parametrized by the number of cycles $N$. As indicated in Fig. 1, the strain at the termination point of unloading at low temperature $T l$ is shown as $\varepsilon_{u}$, the recovery strain by heating under no stress as $\varepsilon_{r}$, the strain recovered by holding at $T_{h}$ for $t_{h}$ as $\varepsilon_{h}$ and the residual strain as $\varepsilon_{p}$. From Fig. 3 it is found that the stress caused by cooling under constant $\varepsilon_{m}$ is considerably larger than the maximum stress at high temperature $T h$. This is due to the thermal stress (tensile stress) caused by the contraction property of the material in relation to temperature decline under the constraint of deformation.

It is noted in Fig. 3 that $\varepsilon_{w}$ is nearly equal to $\varepsilon_{m}$, does not vary with cycling and takes an almost constant value. This corresponds to the large modulus of elasticity at low temperature, and indicates that the variation in modulus of elasticity with cycling is small. For this reason, in many applications of SMP elements, deformation is given in advance at high temperature, and this shape is fixed at low temperature and used. In this case, it is desirable that the shape obtained with loading at high temperature coincide with the shape at low temperature. From the fact that $\varepsilon_{u}$ is nearly equal to $\varepsilon_{m}$ and does not vary with cycling, as noted in Fig. 3, the shape fixity of this material is verified to be very stable against thermomechanical cycling.

From Fig. 3, it is also revealed that with the increase in $N, \varepsilon_{p}$ increases, the recovery strain decreases and the slope of the stress-strain curve in the loading process at high temperature $T_{h}$ becomes large. Every quantity varies significantly in the early cycles and the variation for $N \geqq 5$ becomes small. Also the result that with cycling, the residual strain increases and the recovery strain decreases is identical with the case of shape memory alloys[6].

Figure 4 shows the stress-strain curves obtained from experiments with loading at low temperature for the different number of cycles $N$. As shown in Fig. 2, the strain at the unloading finish point at the low temperature $T i$ is now expressed by $\varepsilon_{\text {, the }}$ strain recovered by heating under no stress by $\varepsilon_{i}$, the strain recovered by holding for 5 minutes at the high temperature $T_{\hbar}$ by $\varepsilon_{h}$, and the residual strain at the finish of cycling by ep. Main points notable from Fig. 4 are as follows. 


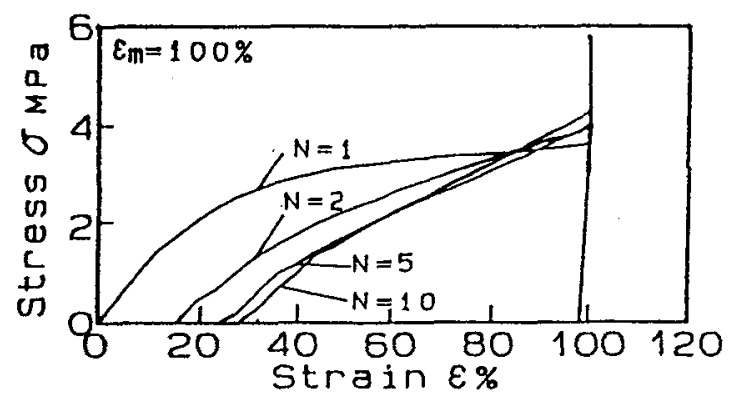

(a) Material HE

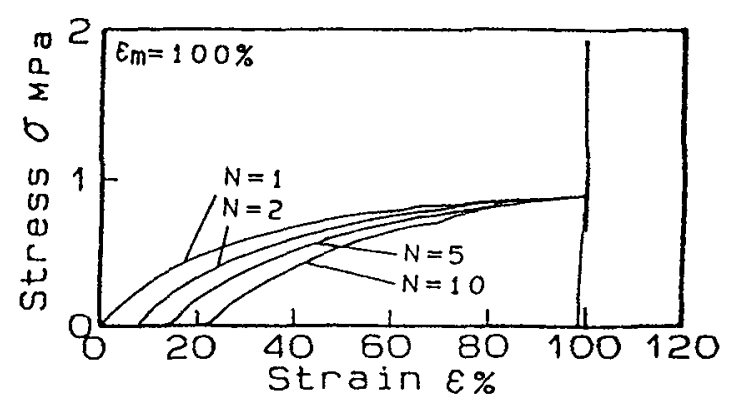

(b) Material LE

Fig.3 Stress-strain curves in the test with loading at high temperature

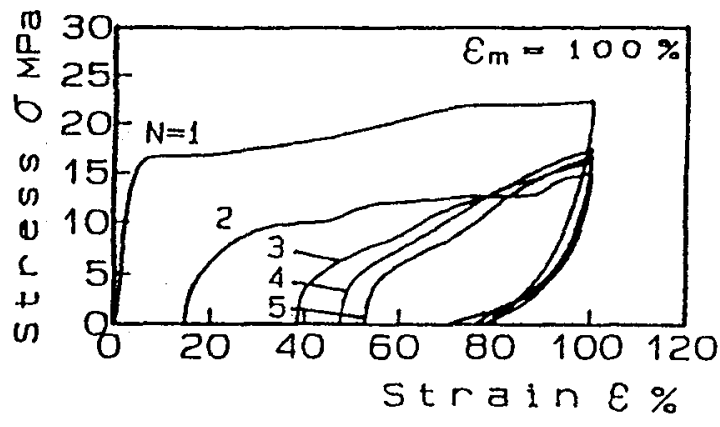

(a) Material $\mathrm{HE}$

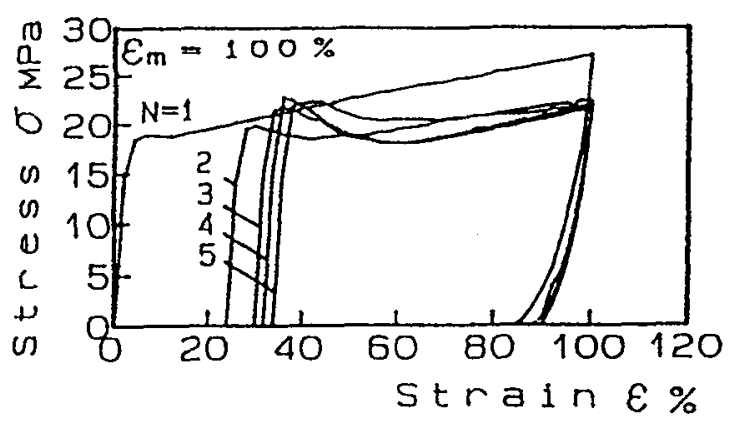

(b) Material LE

Fig.4 Stress-strain curves in the test with loading at low temperature

(1) At the number of cycles $N=1$, the stress for both materials increases almost linearly up to about $15 \mathrm{MPa}$ and thereafter it becomes almost constant because of yielding. The start of this yield corresponds to the creation of constriction in the specimen and the horizontal yield stage in the stress-strain curve corresponds to the development of this constriction. When the strain exceeds about $20 \%$, the stress is slowly elevated by the work-hardening. Also, irrecoverable strain for both materials increases due to the cycling. The rate of increase in $\varepsilon_{p}$ is higher in the early stage of cycling.

(2) The yield stress of material HE largely drops between $N=1$ and $N=2$ in particular, while the irrecoverable strain increases. This is assumed to occur because the entanglement of molecular chains is broken by the first tensile deformation causing slip, and the crystal part (hard segment) is broken and unfolded into the amorphous part (soft segment).

(3) In the unloading process, a large recovery strain is produced in the range of small stress. The strain recovered by unloading is about $10 \sim 20 \%$ of $\varepsilon_{m}$. This substantially differs from the strain obtained with loading at high temperature and unloading at low temperature, which nearly equals $\varepsilon_{m}$ as shown in Fig. 3. 


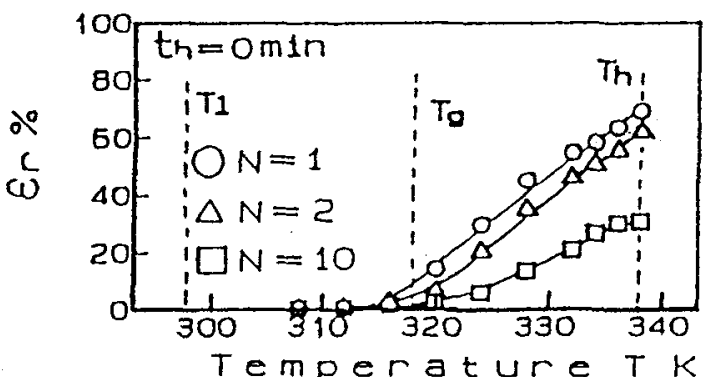

Fig. 5 Dependence of recovery strain on temperature during the heating process in the test with loading at high temperature (Material LE, $\varepsilon_{m}=100 \%$ )

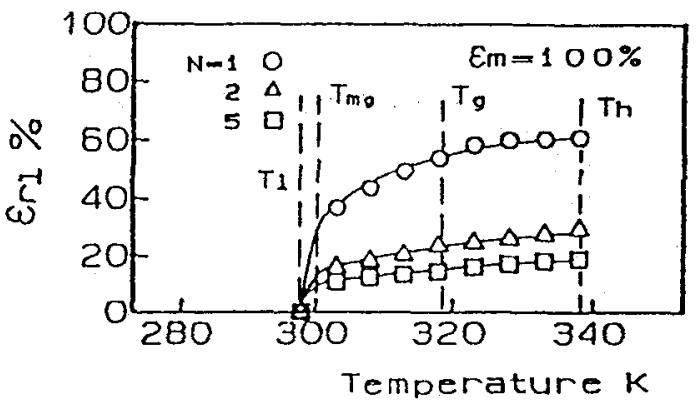

Fig.6 Dependence of recovery strain on temperature during the heating process in the test with loading at low temperature (Material HE, $a_{m}=100 \%$ )

\subsection{Strain Recovery by Heating}

The relationship between the recovery strain $\varepsilon_{r}$ and temperature $T$ in the test with loading at high temperature is shown for the case of material LE, $\varepsilon_{m}=100 \%$ and $t_{h}=0 \mathrm{~min}$ in Fig. 5 . This figure shows the recovery strain during the heating process under no-load. As seen in Fig. $5, \varepsilon_{t}$ is small below $T_{8}=318 \mathrm{~K}$ and increases in proportion to $T$ above $T_{g}$. The reason why $\varepsilon_{8}$ is large above $T_{g}$ is as follows. Recovery deformation of SMP is caused due to micro-Brownian motion of the molecular chain and the motion is activated above $T_{\boldsymbol{g}}$, yielding large amount of $\varepsilon_{i l}[7]$.

The strain-temperature curves moves to the right with an increase in $N$. This means that delay of recovery deformation appears in the application of SMA element. This trend is the same as rise in the transformation temperature with an increase in $N$ for shape memory alloy[6].

Figure 6 shows the relationship between the strain 8 r recovered during the heating process under no stress after unloading and temperature $r$ for Material HE in the test with loading at low temperature. It can be seen from Fig. 6 that the recovery strain $\varepsilon_{l}$ rapidly increases following the start of heating $\left(T_{l}=298 \mathrm{~K}\right)$ and the variation in $\varepsilon \cdot$ is reduced at the temperature of $T_{l}+5 \mathrm{~K}$ or above. It means that most of the recovery strain up to $T_{h}=338 \mathrm{~K}$ arises at the temperature of $303 \mathrm{~K}$ or below. This is substantially different from loading at the high temperature $T k$ where a large recovery strain appears after reaching $T_{8}=318 \mathrm{~K}$ as shown in Fig. 5 . As one of its causes, a drop of $T_{g}$ due to the deformation can be conceived. In order to confirm this, the midpoint glass transition temperature $T m$ was measured by the differential scanning calorimeter (DSC). As a result, $T_{m g}$ of the specimen before starting the test was about $301 \mathrm{~K}$ and that of the specimen after finishing the cyclic test was about $300 \mathrm{~K}$. It is evident from this that the drop of $T_{\mathrm{mg}}$ due to cyclic deformation is extremely small and the recovery strain arising during the heating process is not caused by drop of $T_{g}$. This suggests that with low temperature loading, a large internal stress is produced after unloading owing to the deformation caused by the high stress, and when the heating temperature reaches $T_{m g}$, the strain is rapidly recovered by the action of the internal stress as well as by microscopic Brownian motion of soft segment. 


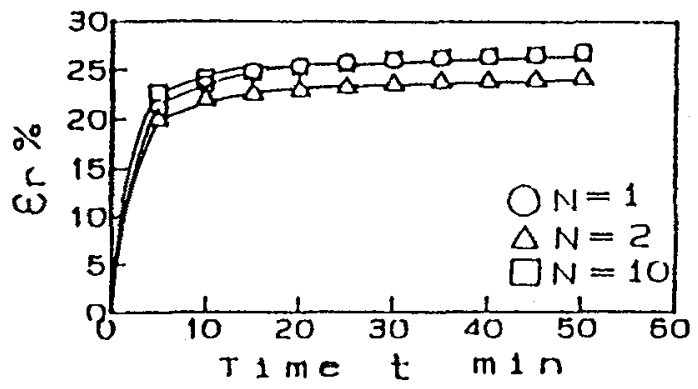

Fig. 7 Dependence of recovery strain on time during the heating process at $T_{h}$ in the test with loading at high temperature (Material LE, $\varepsilon_{m}=100 \%$ )

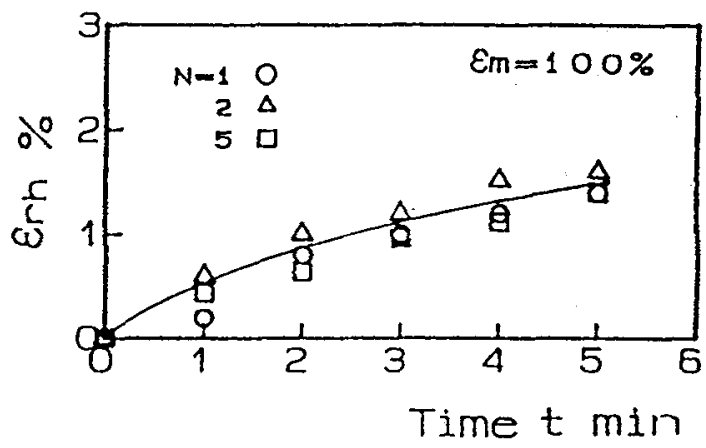

Fig. 8 Dependence of recovery strain on time during the heating process at $T h$ in the test with loading at low temperature (Material HE, $\varepsilon_{m}=100 \%$ )

\subsection{Strain Recovery at High Temperature}

The relationship between the recovery strain $\varepsilon_{h}$ and time $t$ in the test with loading at high temperature is shown for the case of material LE, $\varepsilon_{m}=100 \%$ and $t_{h}=50 \mathrm{~min}$ in Fig. 7 . This figure shows the recovery strain during the holding process at $T_{h}$ under no-load. As seen in Fig. 7, $\varepsilon_{h}$ recovers significantly in the early time and varies slightly after that. Therefore, in order to obtain uniform cyclic recovery deformation, it is better to hold a certain time at high temperature.

Figure 8 shows a relation between the recovery strain 8 and time $t$ in the test with loading at low temperature. It can be seen from Fig. 8 that $\varepsilon_{r}$ increases with the progress of $t$ but the increment is extremely small, only $2 \%$ or less, even after it is held for 5 minutes. Though this value somewhat differs according to the number of cycles $N$, its difference is very small. This defers substantially from the case where $\varepsilon_{n}$ of about $20 \%$ appears in the test with loading at the high temperature $T_{h}$ as shown in Fig. 7. This seems to occur because, in case of loading at low temperature, a sufficient recovery strain arises from the action of internal stress in the heating process.

\section{APPLICATIONS}

SMPs of the polyurethane series have been used in several fields of practical applications. The applications according to the function of the materials are divided into the following classes.

\subsection{Use of Variation in Modulus of Elasticity}

One of applications to use the large amount of variation in modulus of elasticity depending on temperature is a temperature sensor. In the application, the external bias force is combined. As a typical example of application, an autochoke for engine is shown in Fig. 9. Another example of application using the same function is a medical catheter. Because SMP of the polyurethane series has a favorable biological 


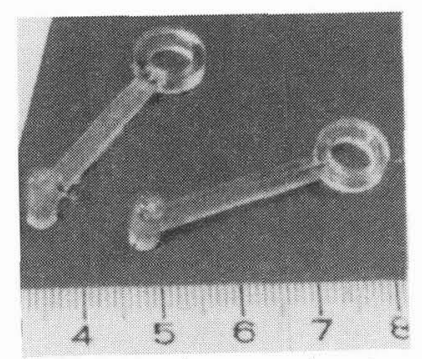

Fig.9 Autochoke for engine

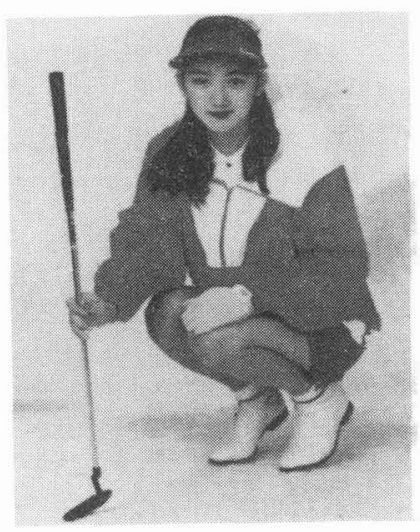

Fig.11 Sportswear
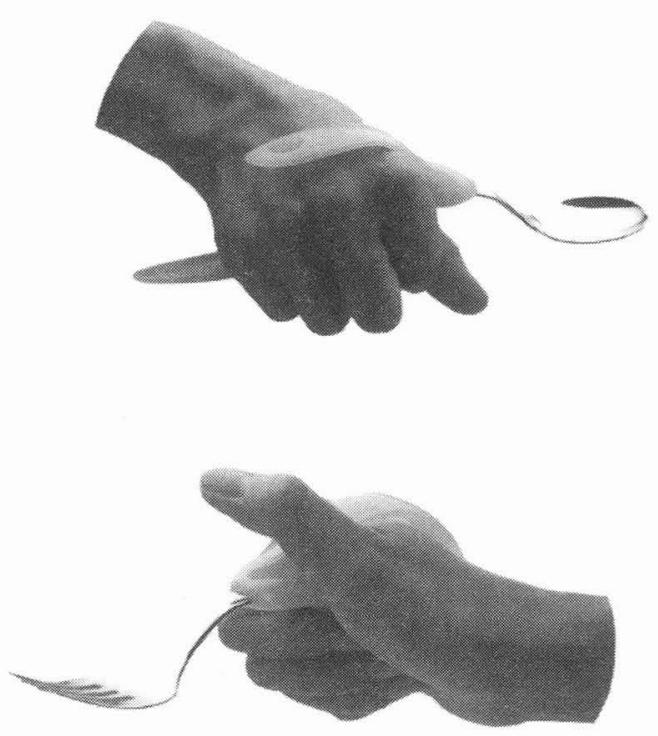

Fig. 10 Spoon and fork handles for handicapped

adaptability owing to its flexibility and antithrombotic nature, development of the catheter is expected.

\subsection{Use of Shape Fixity and Shape Recoverability}

One of the typical application of shape fixity and shape recoverability is a spoon or fork handle for a handicapped person as shown in Fig. 10. With respect to the spoon or fork handle, it is deformed by fitting the form of hand of each person at high temperature and is fixed as its shape at low temperature. The person without grasping power can use the spoon or fork. Another applications are the shape memory straws and films.

\subsection{Use of other Functions}

Humidity permeability, volume expansion, damping property and refractive index vary significantly below and above $T_{z}$ according to the difference of the kinetic property of the molecular chain between below and above $T_{8}$. The applications using the properties are under development. One of the applications of humidity permeability is a sportswear shown in Fig. 11. For the wear, the heat retaining property at low temperature and gas permeability at high temperature are excellent. yielding high quality for the sportswear. 


\section{CONCLUSIONS}

The deformation properties of SMP of the polyurethane series subjected to thermomechanical cycling were investigated. The results are summarized as follows. The deformation properties vary significantly in the early cycles. In the application of SMP elements, mechanical training prior to practical use is effective to the stable cyclic property. For the shape fixity and shape recoverability, the loading at high temperature is superior to the loading at low temperature.

\section{Acknowledgments}

The experimental work in this study was carried out with the assistance of the students of Aichi Institute of Technology, to whom the authors wish to express their gratitude. The authors also wish to express their gratitude to the Scientific Foundation of the Japanese Ministry of Education, Science and Culture for financial support.

\section{References}

[1] Hayashi S, Fujimura $\mathrm{H}$ and Tamura Y, Proc 2nd Japan Int SAMPE, (1991), 76.

[2] Liang C, Rogers CA and Malafeew E, AD-24/AMD-123, Smart Structures and Materials, ASME (1991), 97.

[3] Hayashi S, Ishikawa $N$ and Jiordano C, J Coated Fabrics, 23(1992), 74.

[4] Tobushi H, Hayashi S and Kojima S, JSME Int J, I, 35(3) (1992), 296.

[5] Tobushi H, Hayashi S and Kojima S, Proc SEM Spring Conf Exp Mech, (1993), 520.

[6] Tobushi H, Iwanaga H, Tanaka K, Hor i T and Sawada T, Continuum Mech Thermodyn, 3(1991), 79.

[7] Quay JR, Blackwel1 J and Lee CD, J Macromol Sci-Phys, B, (1986), 61. 